\title{
MUSCLES ACTIVATION DURING FORWARD LUNGE
}

\author{
Mohd Yusof Baharuddin, Ng Woon Shih, Chan Ting Kai, Jia Chengli \& Fazri Wasli
}

Faculty of Sports Science and Coaching, Universiti Pendidikan Sultan Idris, 35900 Tanjung Malim, Perak, Malaysia

Email: yusof.b@fsskj.upsi.edu.my

\begin{abstract}
Journal of Sports Science and Physical Education 7(1): Received: 10 January 2018, Accepted: 15 February 2018 - The purpose of this study was to investigate the muscle activation of the quadriceps and the hamstrings muscle during forward lunge. The study was performed using wireless electromyography (EMG) sensor placed on the interest muscles. The parameter used was the root mean square value during forward lunges activity. The results demonstrated that the rectus femoris muscle was more dominant than the biceps femoris muscle during the initial movement when the knee was in flexion direction and the feet was in dorsiflexion. A linear relationship was initiated between the raw data and RMS value of the EMG signal of the rectus femoris and biceps femoris. The motions elicit eccentric activations of the hamstring muscle groups during lunge activity.
\end{abstract}

Keywords: electromyography, forward lunge, rectus femoris, biceps femoris, muscle activation

\section{INTRODUCTION}

The forward lunge is regularly used in badminton and is recognized for the high physical demands it places on the lower limbs. Despite its common occurrence, little information is available on the biomechanics of lunging in the singles game. A video-based pilot study confirmed the relatively high frequency of lunging, $15 \%$ of all movements, in competitive singles games (Kuntze, Mansfield, \& Sellers, 2010). A lunge is a single-leg bodyweight exercise that works your hips, glutes, quads, hamstrings, core, and the hard-to-reach muscles of the inner thigh. The forward lunge is a common rehabilitation exercise that simulates many activities of daily living. Clinically, variations of the forward lunge exercise are implemented with the intent to target certain muscle groups, depending on an individual's needs (Farrokhi et al., 2008). Forward lunge is the most common exercise to build the lower extremities strength. The muscle involved during the forward lunge is the most used muscle in our daily life activity like climbing up the staircase, walking up on the incline surface, tie your shoes; which are quadriceps, hamstring, and gluteus. The main aim of this study was to determine which muscle is mainly used in performing lunge either rectus femoris muscle (front muscle of thigh) or biceps femoris muscle (back muscle of thigh). 


\section{METHODOLOGY}

\section{Participants}

The subject was a university badminton team male single player, with height of $177 \mathrm{~cm}$, weight $70 \mathrm{~kg}$, and age 21 years old. He was healthy at the time of testing, which was operationally defined as no history of previous ankle, knee, hip, or back musculoskeletal conditions that could influence his ability to perform the forward lunge. The participant was actively involved in badminton training and physical training. The participant is a right-handed player while playing his sport. The participant was given a verbal description of the study procedure, the opportunity to ask questions and the demonstration on how to perform the forward lunge.

\section{Experiment design}

The sensors of the EMG were located at the muscle that will be tested. A sensor was attached at the middle of the quadriceps which is the rectus femoris using a tape. Another sensor was pasted at the posterior side of the thigh muscle which located at the bicep femoris muscle. The rectus femoris was located at the middle of the thigh while the bicep femoris muscle was located at the middle of the posterior side of the thigh as showed in Figure 1.
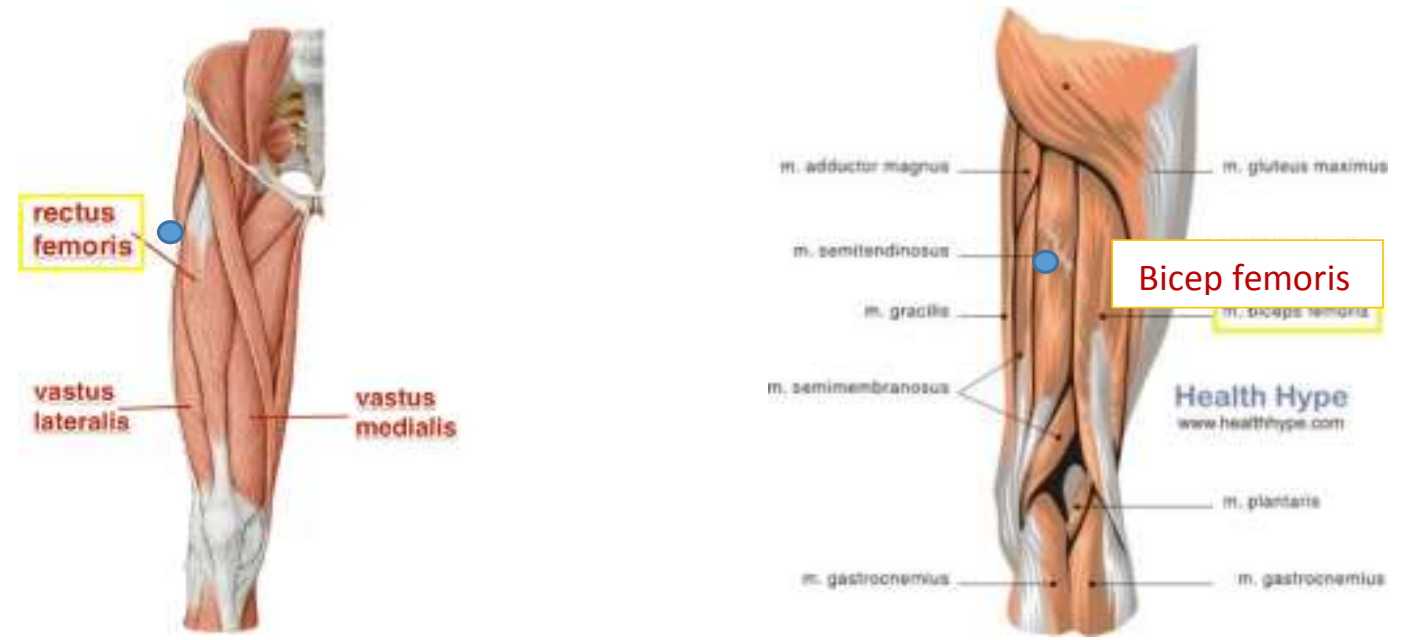

Figure 1 Muscle interest in forward lunge

\section{Procedure}

The participant wore comfortable sport attire with short pants and was barefoot during the data collection. The participant's dominant leg (a more used leg for moving during the badminton game) was used as the stepping limb in the forward lunge motion. He stood in an anatomical position and the photos of the anterior and posterior view of the lower limbs were taken using camera to make sure the participant has a healthy standing posture. After checking all the body 
posture, the sensors on the leg was checked and switched on to make sure the sensor works. Usually the dominant limb was determined by kicking a ball. The dominant limb for a badminton player can be defined as the preferred limb to move anteriorly and bend the knee. A proper forward lunge can be standardized by few important factors. Firstly, when the participant was instructed to start the exercise, the participant has to step his dominant leg forward and make sure the eye is staring in front, the body (core) must be maintained erected and perpendicular to the floor. Once the participant reached the point of forward lunge (the knee in front must be either in right angle $90^{\circ}$ or less than $90^{\circ}$, the limb behind must be fully stretched until the limit, by checking this, make sure the angle of the knee as largest as possible), he was instructed to immediately push backward through the dominant limb and return to the full standing starting position. The tester stood beside the participant to give the instruction of 5 repetitions. The EMG sensors is put at the middle of quadriceps rectus femoris and hamstring biceps femoris using tapes as showed in Figure 2.
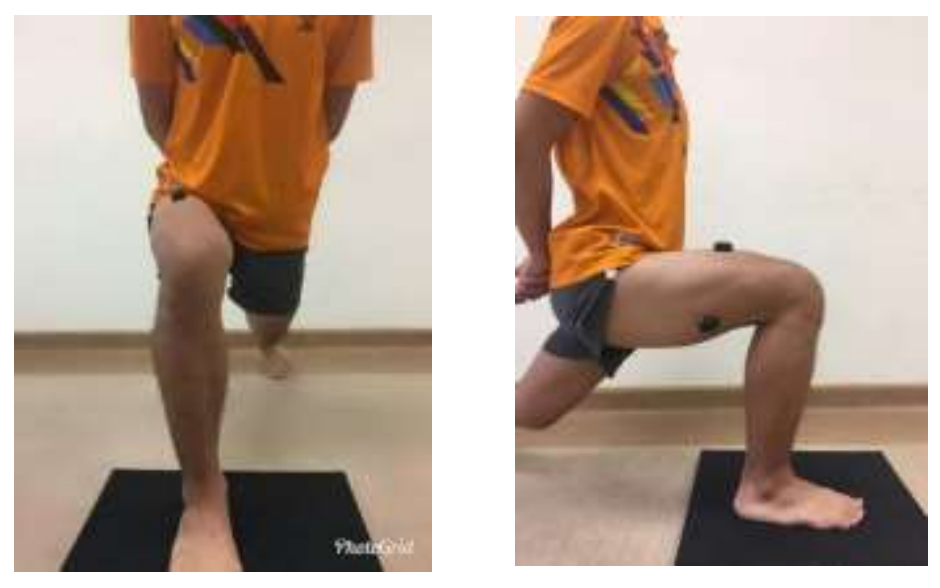

Figure 2 Demonstration of forward lunge (anterior view) and (side view)

\section{Test protocol}

The subject is required to do 5 times of the forward lunge for the right limb. The subject need to keep the upper body straight, with shoulders back and relaxed and chin up (pick a point to stare at in front so he don't keep looking down). He need to always engage hiscore and step forward with one leg, lowering the hips until both knees are bent at about a 90-degree angle. The front knee should be directly above the ankle, not pushed out too far, and the other knee doesn't touch the floor. The subject needs to keep the weight in his heels as he push back up to the starting position.

\section{Data Acquisition}

The electrical impulses (action potentials) are transferred to the different muscles in every part of our body when the contraction of muscle is happening. Action potentials (those electrical 
impulses that send signals around your body) are nothing more than a temporary shift (from negative to positive) in the neuron's membrane potential caused by ions suddenly flowing in and out of the neuron. And this action potentials cause the muscle to contract when they reach the neuromuscular junction. The EMG system was used to determine the amount of the electric signal produced during the contraction of the muscle.
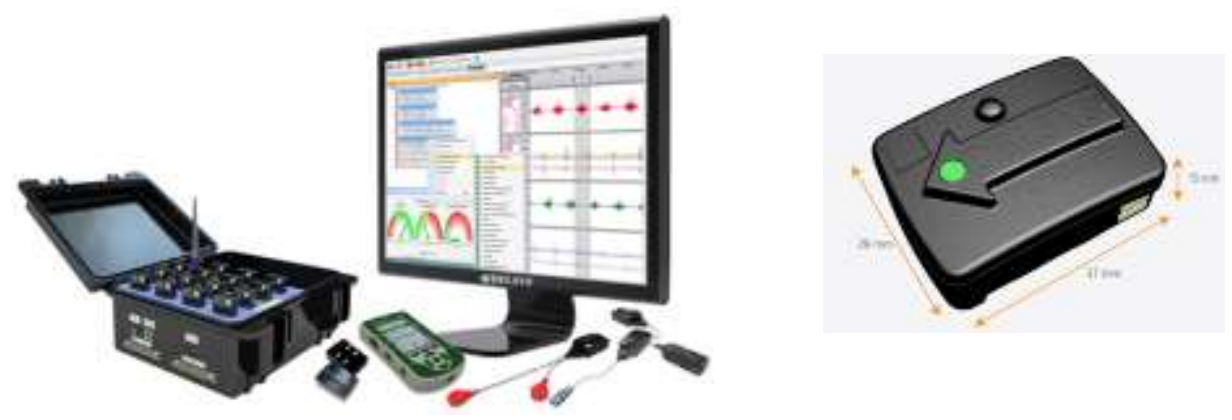

Figure 3 Trigno Delsys Wireless EMG system

Trigno Wireless System is a high-performing device designed to make EMG signal detection reliable and easy as showed in Figure 3. The system is capable of streaming data digitally into EMGworks software or 3rd party software, or via analog channels for integration with motion capture and other 3rd party data acquisition systems. Full triggering features further expand the possibility for integration with additional measurement technologies. The Trigno Flex sensor is an EMG biofeedback sensor designed to be used exclusively as a component of the Trigno EMG System. The sensor is designed to accommodate research needs requiring an expanded bandwidth range $(10-850 \mathrm{~Hz})$ or an expanded amplitude input range $(22 \mathrm{mV} \mathrm{rti})$. The original recommended parameters of the standard sensor $(20-450 \mathrm{~Hz}, 11 \mathrm{mV}$ rti range) are still available as well.

\section{Data Processing}

The voltage of the electrical signal produced by the muscle was recorded with time using the EMG system. This allowed us to evaluate the amount of the electrical signal produced by the two different muscle during the forward lunges was done by the participant. By using the raw data, we first plot a graph and make a comparison between the rectus femoris and biceps femoris muscle. After that, we used the Root Mean Square (RMS) value to plot another graph. The root-mean-square (RMS) is not a statistic you hear too much about, because it is mostly used as a part of other statistics, such as the standard deviation, which are much more famous. The root mean square is a measure of the magnitude of a set of numbers. It gives a sense for the typical size of the numbers. 


\section{Root mean square}

The root-mean-square (RMS) envelope of the EMG signal is calculated using a moving window, with each window of data calculated according to the following equation:

$$
R M S=\left(\frac{1}{s} \sum_{1}^{S} f^{2}(s)\right)^{\frac{1}{2}}
$$

where $\mathrm{S}$ equals the window length (points) and $\mathrm{f}(\mathrm{s})$ equals the data within the window. The RMS calculation is considered to provide the most insight on the amplitude of the EMG signal since it gives a measure of the power of the signal, while producing a waveform that is easily analyzable.

\section{RESULTS}

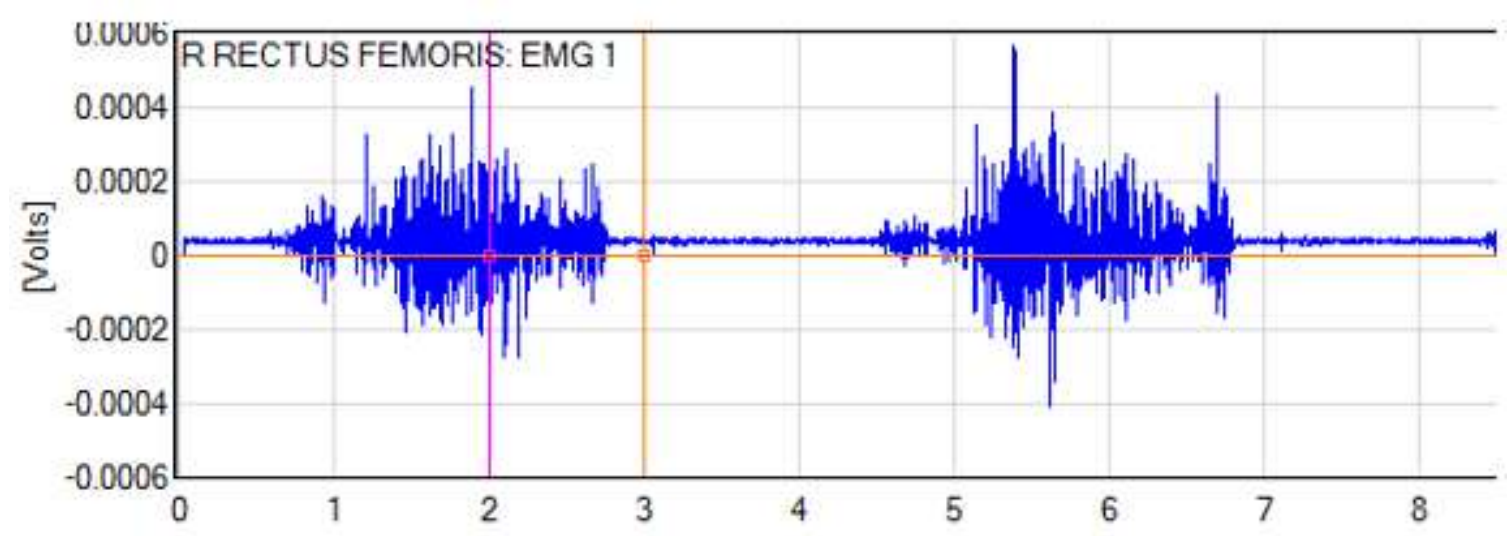

Figure 4 Raw data for rectus femoris muscle activation

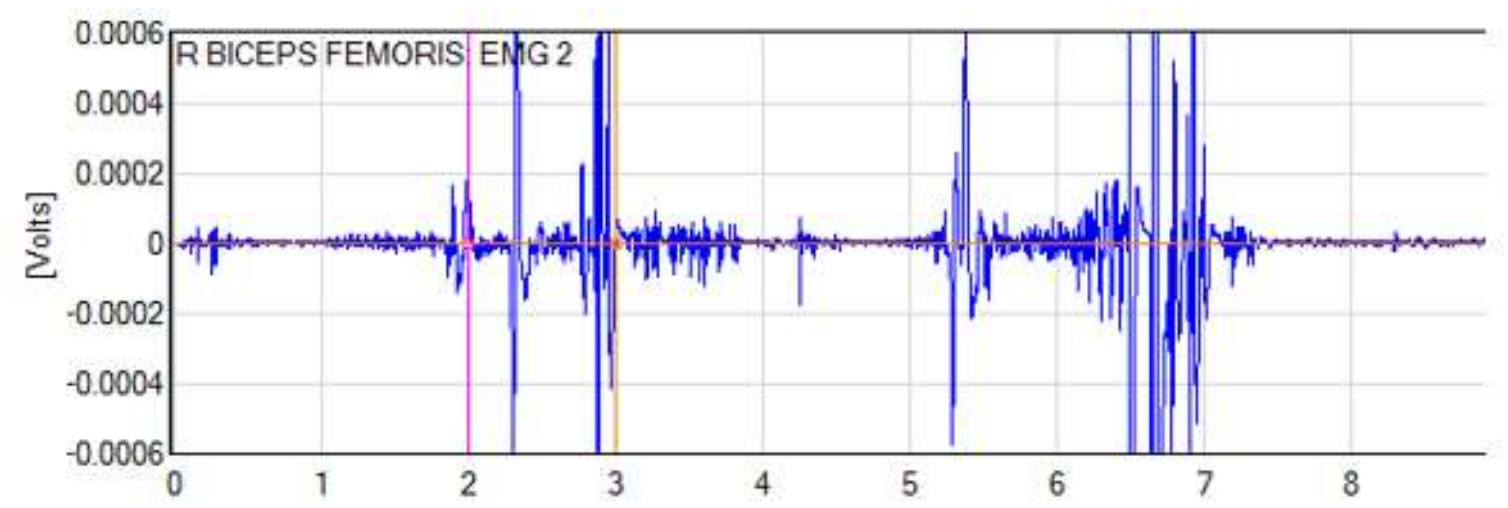

Figure 5 Raw data for biceps femoris muscle activation

Figure 4 and Figure 5 is representing the contraction of rectus femoris and bicep femoris. From Figure 4, we can see that during the stage before $1 \mathrm{~s}$ and the stage before $3 \mathrm{~s}$, the electrical signal produced by the rectus femoris muscle was significantly high compared to the electrical 
signal of the stage between $3 \mathrm{~s}$ and $4 \mathrm{~s}$. This is because during the $1 \mathrm{~s}$ and $3 \mathrm{~s}$, the forward lunge was started to perform, the leg was moving forward and tend to bend the knee. The rectus femoris muscle was fully tensed during the knee bending motion. From Figure 5, we noticed that during the $3 \mathrm{~s}$, that was a high voltage of electrical signal produced. So, there was a huge contraction of biceps femoris muscle during the $3 \mathrm{~s}$. By comparing both graph the phenomenon showing us that the biceps femoris muscle works right after the rectus femoris works.

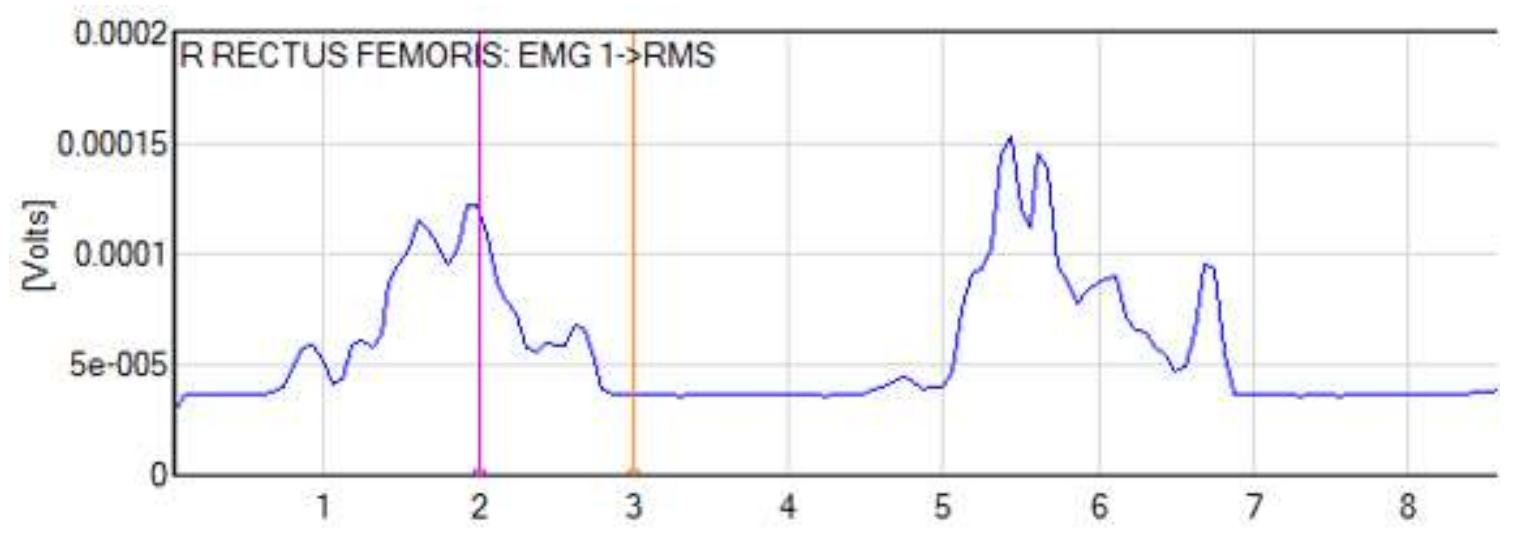

Figure 6 RMS graph of rectus femoris

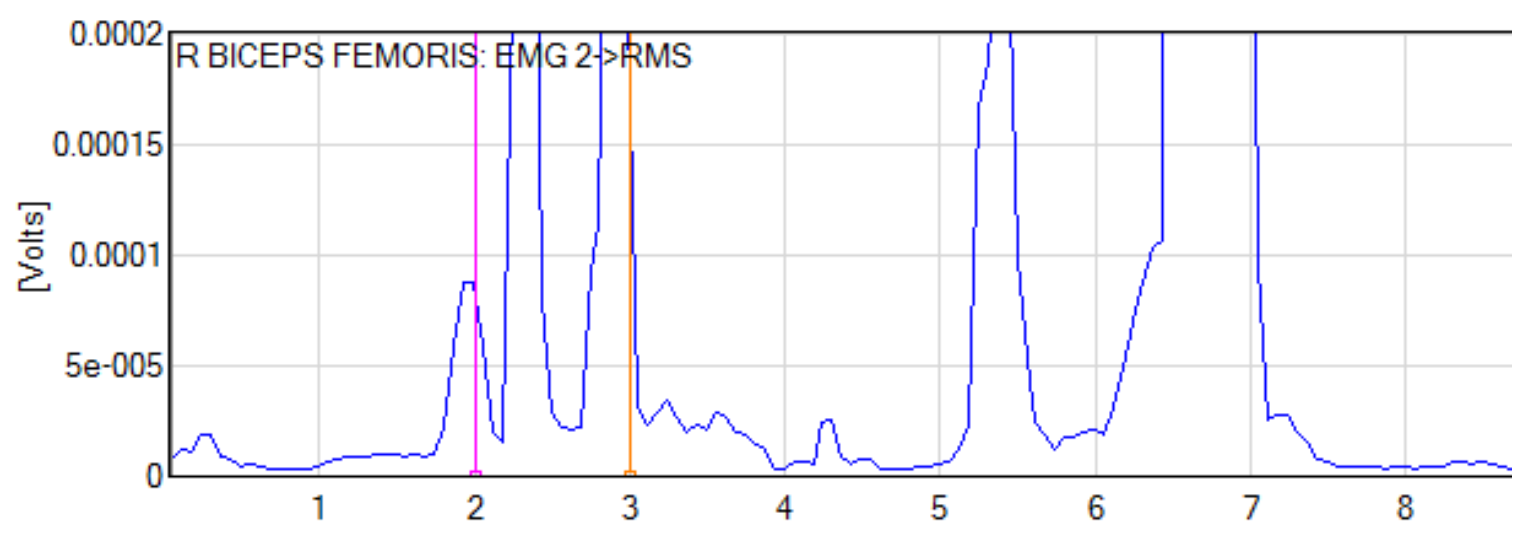

Figure 7 RMS graph of biceps femoris

Figure 6 and Figure 7 are the graph plotted using the processed data. The raw data was processed into the RMS value. This is to make all the value to be positive and it's easier to see the variety of the voltage with time. By comparing both as showed in Figure 8, we can clearly noticed that, during the $3 \mathrm{~s}$, the bicep femoris had a significantly high value of voltage, and during $1 \mathrm{~s}$ to before $3 \mathrm{~s}$, the rectus femoris muscle produced electrical signal. We can conclude that, the bicep femoris contract right after the rectus femoris contract. 


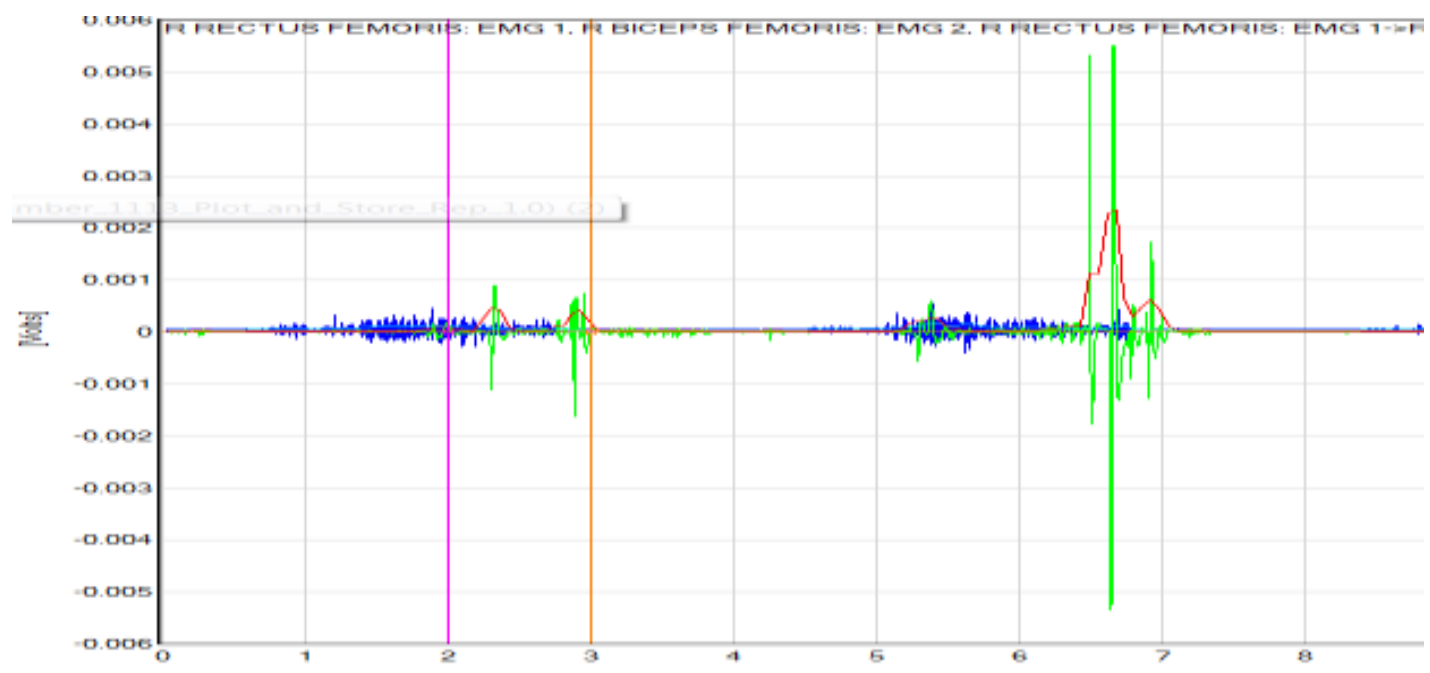

Figure 8 Overlaid graph of all raw and processed data (RMS) for both muscles

\section{DISCUSSION}

Since the rectus femoris muscle used more during the initial movement when the knee was bending down and the feet is going forward and touch the floor. The flexion of knee caused this happened. So, we can say that the rectus femoris muscle act as an agonist muscle and the biceps femoris muscle act as an antagonist muscle when performing the flexion of knee. In contrast, the biceps femoris muscle used the most when the forward lunge was going to end and the participant was trying to finish the lunge by extend the knee back to the normal posture. This can be concluded that the biceps femoris muscle act as an agonist muscle and the rectus femoris muscle act as an antagonist muscle during the extension of the knee. We found that the hamstring muscles had a very short and rapid period of elongation during the first part of the walking forward lunge. Being two-joint muscles, the hamstring length depends on both knee and hip joint angles. The angle of the hip has more impact on the length of the hamstrings than the angle of the knee (Hawkins \& Hull, 1990), due to the longer moment arm at the hip, and this relation increases with increasing knee angle. Small changes in the hip angle may compensate for changes in knee joint angle, which may explain why there is an isometric contraction of the hamstrings during the early lunge phase even though the knee flexes at this time. In the rectus femoris, we found two periods during the forward lunge when the muscle elongates while active: at foot strike and at the end of the movement. In the beginning of the foot strike, there is a marked elongation of the rectus femoris, followed by a sustained period of concentric contraction. Because the comparisons were repeated measures on the same subject using the same normalization technique, the comparative analysis of the EMG signals of the muscle is valid. The subjects in this study performed the lunge with a maximal effort. When attempting to use these lunge exercises for training or rehabilitation, the difficulty of forward lunge should be gradually increased (Jönhagen, Ackermann, \& Saartok, 2009). 


\section{Conclusion}

The rectus femoris muscle act as an agonist muscle and the biceps femoris muscle act as an antagonist muscle when performing the flexion of knee. The biceps femoris muscle act as an agonist muscle and the rectus femoris muscle act as an antagonist muscle during the extension of the knee.

\section{References}

Farrokhi, S., Pollard, C. D., Souza, R. B., Chen, Y. J., Reischl, S., \& Powers, C. M. (2008). Trunk position influences the kinematics, kinetics, and muscle activity of the lead lower extremity during the forward lunge exercise. Journal of Orthopaedic \& Sports Physical Therapy, 38(7), 403-409.

Hawkins, D., \& Hull, M. (1990). A method for determining lower extremity muscle-tendon lengths during flexion/extension movements. Journal of Biomechanics, 23(5), 487-494.

Jönhagen, S., Ackermann, P., \& Saartok, T. (2009). Forward lunge: a training study of eccentric exercises of the lower limbs. The Journal of Strength \& Conditioning Research, 23(3), 972-978.

Kuntze, G., Mansfield, N., \& Sellers, W. (2010). A biomechanical analysis of common lunge tasks in badminton. Journal of Sports Sciences, 28(2), 183-191.

$\equiv$ Mohd Yusof Baharuddin

Faculty of Sports Science and Coaching,

Universiti Pendidikan Sultan Idris,

35900 Tg Malim, Perak,

Malaysia 